\title{
Guest Editorial: Data Fusion, Integration and Advances of Non-Destructive Testing Methods in Civil and Environmental Engineering
}

Fabio Tosti ${ }^{*}$, Andrea Benedetto ${ }^{2}$, Luca Bianchini Ciampoli ${ }^{2}$, Fabrizio D'Amico ${ }^{2}$, Christina Plati ${ }^{3}$ and Andreas Loizos $^{3}$

\author{
${ }^{1}$ School of Computing and Engineering, University of West London (UWL), St Mary's Road, Ealing, London W5 5RF, United Kingdom \\ (Fabio.Tosti@uwl.ac.uk) \\ 2Department of Engineering, Roma Tre University, Via Vito Volterra 62, 00146, Rome, Italy. (andrea.benedetto@uniroma3.it; \\ luca.bianchiniciampoli@uniroma3.it; fabrizio.damico@uniroma3.it) \\ ${ }^{3}$ Laboratory of Pavement Engineering, National Technical University of Athens (NTUA), Zografou Campus, 9, Iroon Polytechniou Str., \\ Zografou, 15780 Athens, Greece (cplati@central.ntua.gr; aloizos@central.ntua.gr) \\ *Corresponding Author
}

ORCID ID: Fabio Tosti (0000-0003-0291-9937); Andrea Benedetto (0000-0002-1006-8928); Luca Bianchini Ciampoli (0000-0003-09812104); Fabrizio D’Amico (0000-0003-1581-6320); Christina Plati (0000-0002-2452-0233); Andreas Loizos (0000-0001-7533-5233)

Use of non-destructive testing (NDT) methods has tremendously increased over the last decades in a wide range of civil and environmental engineering applications. Research on stand-alone use of electric, electromagnetic, optical and acoustic NDT techniques has rapidly progressed and potentials of these methods have been comprehensively explored and evaluated, making the majority of techniques established in many areas of endeavour. Research focus areas have been mostly on developing new theories, the advancement of hardware and software components, and on introducing novel surveying protocols, data processing and interpretation methods. Accordingly, the standard of quality and accuracy of data has reached very high levels with available technology.

Parallel to this, the integration between sensing technologies, in terms of collecting and modelling multisource, multi-scale and multi-temporal data, has become a very challenging task in research. This concept is in fact being acknowledged as a fundamental area of research development that could highly contribute towards the enhancement of the capabilities of existing NDT methods in new and non-conventional scenarios. This trend is motivated by the need to provide more effective solutions for the investigation of complex scenarios. At the same time, a balance among project costs and time requirements on one hand, and resource management-related constraints due to the involvement of multiple methods, equipment and interdisciplinary expertise in the other, must be maintained.

The papers presented in this Special Issue stem from the organisation of a Session on "Data Fusion, Integration, Correlation and Advances of Non-destructive Testing Methods and Numerical Developments for Engineering and Geosciences Applications" held at the European Geosciences Union (EGU) General Assembly in Vienna, Austria, from 7 to 12 April 2019. The Session was aimed to collect the latest research contributions in the areas of NDT methods, theories and new developments for applications in civil and environmental engineering. Special research focus areas were: i) the development of novel data-fusion and correlation approaches and ii) data-integration methodologies, and iii) the advances achieved in the use of stand-alone NDT technologies.

The first topical area incorporated civil and environmental engineering research on data-fusion and correlation of multi-source information from different NDT methods and techniques, as well as on the datafusion of multi-scale information from single non-destructive sensing technologies. The area related to the development of novel data-integration methodologies included research on the incorporation of multisensing, multi-scale and multi-temporal techniques for structural health monitoring and investigation of 
historical bridges and transport infrastructures, as well as the integration of information from simulation and real-life domains of analysis for pavement engineering applications. The last topical area was focussed on presenting latest research advances from the stand-alone use of the ground-penetrating radar (GPR) technology in areas concerned with railway engineering and conservation.

In the first Section of this Special Issue, Maroti et al. [1] provide a multi-source data-fusion approach combining different NDT techniques, i.e., three-dimensional surface and volumetric digital imaging techniques, as well as position-resolved element composition analysis on echinoid fossils. To elaborate, the joint application of structured-light (SL) optical scanning, neutron tomography (NT) and position-sensitive prompt gamma neutron activation analysis (PGAA) has proven its viability at providing effective morphological and compositional analyses of fossils. This contributes to form the essential knowledge about the classification and development of the species as well as about the environmental aspects during and after the life of the exemplar. Plati et al. [2] report a data-correlation approach incorporating multi-source information from deflection-based and electromagnetic non-destructive methods for the assessment of road pavements. The focus of the research is to investigate the correlation between data collected on road flexible pavements with the falling weight deflectometer (FWD) and the GPR techniques in order to estimate the thickness of the asphalt concrete (AC) layer directly from FWD deflection parameters. The results demonstrate that FWD allows to evaluate pavement geometric features with high accuracy. Bi et al. [3] present a data-fusion approach based on signal processing of multi-frequency GPR datasets. The aim is to compensate between signal resolution and penetration features, whilst providing a more comprehensive and high-resolution detection of both shallow and deep targets. To this effect, the authors propose the "timedomain fusion with/without weights" and the "frequency-domain fusion with weights" algorithms, applied to $900 \mathrm{MHz}, 1600 \mathrm{MHz}$ and $2600 \mathrm{MHz}$ central-frequency data. The approach is tested in numerical and real domains of investigation (both laboratory and real-life scenarios) and it is successfully proven to work for the creation of single GPR profiles incorporating multi-scale information from low to high frequencies.

The second section of this Special Issue presents research around the subject area of developing novel dataintegration methodologies for the investigation of civil engineering structures and infrastructures using multiple or stand-alone NDT methods. D'Amico et al. [4] report the integration between satellite remote sensing and ground-based NDT techniques for structural health monitoring of railway infrastructures. To elaborate, information collected with the interferometric synthetic aperture radar (InSAR) and the GPR techniques are integrated for monitoring the structural integrity of the rail-abutment transition area in railway bridges. The results demonstrate the viability of the proposed integrated approach. On one hand, GPR is proven effective to obtain subsurface structural details of the track-bed as well as to identify construction-related issues. On the other hand, the availability of multi-temporal high-resolution vertical displacement data allows InSAR to monitor successfully subsidence at the rail-abutment transition area. Alani et al. [5] present an integrated investigative approach in health monitoring of historical bridges using GPR and InSAR technologies. This paper provides an overview of the existing health monitoring and assessment methods for masonry arch bridges. A case study is presented on the application of these two techniques to the monitoring of the "Old Bridge" at Aylesford, Kent, UK - a $13^{\text {th }}$ century bridge, crossing the Medway river. GPR has proven essential at providing structural detailing in terms of subsurface geometry of the superstructure as well as the exact positioning of the structural ties. InSAR has identified measures of structural displacements caused by the seasonal variation of the water level in the river and the river bed soil expansions. Biscarini et al. [6] report the cooperative use of NDT techniques for structural and material degradation evaluation of historical bridges. A case study is presented on the integration of multi-source information collected from the use of the unmanned aerial vehicle (UAV) photogrammetry, the infrared thermograph (IT) and the GPR techniques on the Roman masonry arch bridge "Ponte Lucano" in Tivoli, Italy. The results demonstrate consistency between the outputs of these techniques and the ability of these approaches to monitor the state of material degradation and source of defects, as well as to recuperate unique historical information about construction stages and modifications of the bridge structure. In regard 
to the use of stand-alone NDT methods, Rasol et al. [7] propose an experimental and numerical approach for the identification of early cracking in composite rigid pavements using GPR. A comprehensive set of real (both laboratory and real-life scenarios) and numerical investigations are presented. These include, use of vertical and horizontal antenna polarisations over surface cracks propagating vertically down to the underlying structural concrete layer and surface cracks not aligned with cracks at the concrete layer, amongst others. Results from a comparative analysis performed across the experimental and numerical domains of analysis highlight, amongst others, the possibility to detect $10 \mathrm{~mm}$ wide fractures as well as some signal features from the structural concrete layer. Wang et al. [8] report the development of a novel GPR-based signal processing algorithm for real-time monitoring of the density of AC layers in road flexible pavement structures during compaction. First, the viability of the approach is tested in the numerical domain, and it is later validated in lab-controlled and real-life conditions. To elaborate, the "mean reflection coefficient algorithm" and the "digital filter design method" are used to remove the surface moisture and smooth the density profile. Results demonstrate the viability of GPR for utilisation in density monitoring of AC layers. The importance of providing incorporation in the prediction model of accurate dielectric permittivity values for the aggregate types in the AC layer is also proven.

The last section of this Special Issue reports the latest research findings from the stand-alone use of GPR in the areas of railway engineering and conservation. Artagan and Borecky [9] present an experimental study for the investigation of railway ballast materials. A comprehensive amount of GPR tests $(2000 \mathrm{MHz}$ antenna central frequency) performed in the laboratory environment under various fouling and moisture conditions are presented to estimate relative dielectric permittivity trends in granite ballast polluted with three different fouling agents (i.e., sand, gravel and a mixture of them), from dry to saturated moisture conditions. Furthermore, a case study is discussed that considers real-life GPR surveys $(400,900$, and $2000 \mathrm{MHz}$ antenna central frequencies) carried out on the same ballast material type over a track section composed of metal, wooden and concrete sleepers. Results demonstrate the viability of integrating dielectric permittivity information obtained in lab-controlled conditions into data processing algorithms used for the evaluation of the track-bed subsurface structure in real-life surveys. The last two papers report on the use of GPR for the conservation of trees. de Mahieu et al. [10] discuss the performance of two GPR signal processing techniques for assessment of the short-scale variability in physical soil properties potentially involved with regeneration processes of cork oak trees. To this effect, a case study at the Maâmora forest, Morocco, is presented, where two GPR antenna systems are used. First, a newly-developed frequency domain GPR system (range of frequencies: $85 \div 5400 \mathrm{MHz}$ ) equipped with an off-ground antenna is used to provide extensive mapping of the soil surface water content through the "full-wave inversion" technique. A conventional time-domain GPR system equipped with a ground-coupled antenna ( $400 \mathrm{MHz}$ central frequency) is used to collect information about the subsurface structure. Outcomes are therefore compared against dendrometric-performance factors about the regeneration of the trees. Results demonstrate that soil water content is a relevant indicator of successful cork oak regeneration, thereby proving the effectiveness of GPR as a decision support tool for sustainable tree planting. Alani et al. [11] report a novel multi-stage GPR data progressing framework for the assessment and health monitoring of decay in tree trunks. To this effect, a positioning method for an accurate association between single GPR traces and corresponding coordinates along the irregular bark surface is initially discussed. A processing pipeline is therefore presented that aims at eliminating the ringing noise due to the multi-layered internal structure of trees. Finally, a "reverse-time (RT) migration" approach is applied to the processed radargram in order to effectively map the reflectors present within the trunk. The presented data-processing framework is successfully proven to work in both numerical and real-life domains of investigation. In particular, the processed data were mapped against the cut sections of the tree for validity purposes, demonstrating the viability of the approach.

To conclude, the research presented in this Special Issue emphasises the importance of using data-fusion, correlation and integration strategies with the information collected through NDT methods, and proves how incorporating the capabilities of different NDT techniques can provide ample opportunity for the research 
expansion in many areas of endeavour. In this context, it is observed that most research presents new methods and approaches where algorithms are based on data-integration of multi-sensing and multi-scale information, which are often tested across numerical and real (both laboratory and real-life environment) domains. Lower amount of research is instead developed following data-fusion and data-correlation approaches. However, it is believed that future research will be aimed at exploring this particular area of development, in order to improve upon current limitations in established application fields, as well as to investigate new potential avenues for addressing unexplored scenarios. Furthermore, it is worthy to note that GPR is the most popular technique among the ground-based NDT methods used for data-fusion and integration purposes, with specific applications to historical bridges, highway, and railway transport infrastructures. Parallel to this, new research trends are emerging based on the integration of multi-temporal and multi-scale information from satellite remote sensing and ground-based non-destructive techniques. In terms of the application of self-standing NDT methods, GPR is confirmed as the most popular method, contributing to new research findings in niche areas of special environmental focus.

\section{Acknowledgments}

The Guest Editors are sincerely grateful to all the referees for contributing to improve the quality of the research published in this Special Issue. A sincere acknowledgement goes to the Editor-in-Chief of the Journal, Professor Dale E. Chimenti, for the opportunity and support provided across the whole process.

\section{References}

[1] Maróti B, Polonkai B, Szilágyi V, Kis Z, Kasztovszky Z, Szentmiklósi L, Székely B. Joint Application of Structured-Light Optical Scanning, Neutron Tomography and Position-Sensitive Prompt Gamma Activation Analysis for the NonDestructive Structural and Compositional Characterization of Fossil Echinoids. NDTE Int 2020 (This issue).

[2] Plati C, Loizos A, Gkyrtis K. Integration of non-destructive testing methods to assess asphalt pavement thickness. NDTE Int 2020 (This issue).

[3] Bi W, Zhao Y, Shen R, Li B, Hu S, Ge S. Multi-frequency GPR data fusion and its application in NDT. NDTE Int 2020 (This issue).

[4] D'Amico F, Gagliardi V, Bianchini Ciampoli L, Tosti F. Integration of InSAR and GPR techniques for monitoring transition areas in railway bridges. NDTE Int 2020 (This issue).

[5] Alani AM, Tosti F, Bianchini Ciampoli L, Gagliardi V, Benedetto A. An Integrated investigative approach in health monitoring of masonry arch bridges using GPR and InSAR technologies. NDTE Int 2020 (This issue).

[6] Biscarini C, Catapano I, Cavalagli L, Ludeno G, Pepe FA, Ubertini F. UAV Photogrammetry, Infrared Thermography and GPR for Enhancing Structural and Material Degradation Evaluation of the Roman Masonry Bridge of Ponte Lucano in Italy. NDTE Int 2020 (This issue).

[7] Rasol MA, Pérez-Gracia V, Solla M, Pais JC, Fernandes FM, Santos C. An experimental and numerical approach to combine ground penetrating radar and computational modelling for the identification of early cracking in cement concrete pavements. NDTE Int 2020 (This issue).

[8] Wang S, Al-Qadi IL, Cao Q. Factors impacting monitoring asphalt pavement density by ground penetrating radar. NDTE Int 2020 (This issue).

[9] Artagan SS, Borecky V. Advances in the nondestructive condition assessment of railway ballast: a focus on GPR. NDTE Int 2020 (This issue).

[10] de Mahieu A, Ponette Q, Mounir F, Lambot S. Using GPR to analyze regeneration success of cork oaks in the Maâmora forest (Morocco). NDTE Int 2020 (This issue).

[11] Alani AM, Giannakis I, Zou L, Lantini L, Tosti F. Reverse-time migration for evaluating the internal structure of treetrunks using ground-penetrating radar. NDTE Int 2020 (This issue). 